\title{
TARGETED AND NON-TARGETED ANALYSIS BASED ON ULTRA HIGH PERFORMANCE LIQUID CHROMATOGRAPHY AND FOURIER TRANSFORM INFRARED SPECTROSCOPY FOR PARIS SPECIES OF DIFFERENT GEOGRAPHICAL ORIGINS
}

\author{
Yuangui Yang ${ }^{\mathrm{a}}$, Yanli Zhao ${ }^{\mathrm{a}}$, Ji Zhang ${ }^{\mathrm{a}}$, Shaobing Yang*,a and Yuanzhong Wang*,a, (D) \\ Institute of Medicinal Plants, Yunnan Academy of Agricultural Sciences, Kunming 650200, China
}

Recebido em 02/11/2018; aceito em 09/01/2019; publicado na web em 18/03/2019

\begin{abstract}
Various species of Paris have been used in treatments for their antitumor, antimicrobial, hemostatic, anthelmintic and immune-stimulating properties, among others. The bioactive markers of these plants including paris saponins I (PSI), paris saponins II (PSII), paris saponins VI (PSVI) and paris saponins VII (PSVII) are responsible for inhibiting nasopharyngeal carcinoma epithelial cell and ethanol-induced gastric lesions. This paper intends to comprehensively characterize the distribution of bioactive markers and illustrate the relationship between chemical profile and Paris spp.. A targeted quantitative analysis by ultra high performance liquid chromatography (UHPLC) was established for the simultaneous separation and determination of PSI, PSII, PSVI and PSVII. Non-targeted analysis based on Fourier transform infrared (FT-IR) spectroscopy, with the aid of partial least squares discriminant analysis (PLS-DA), was also performed. In addition, similarity analysis combining UHPLC and FT-IR spectroscopy were employed to evaluate Paris of different species and geographical origins. Quantitative analysis indicated that the content of saponins varied markedly, and the average total saponins of Paris axialis (PA) of a specific region (Baoshan) was higher than the other species. In addition, total saponins of P. polyphylla var. yunnanensis (PPY) collected from Xishuangbanna was higher than the same species of other regions. Non-targeted analysis indicated that PA was different from other species and PPY in the Baoshan differed from other regions. The results indicate that comprehensive analyses for geographical origin discrimination of herbal medicines are expected to be employed in further investigations.
\end{abstract}

Keywords: targeted quantitative analysis; non-targeted analysis; UHPLC-UV; FT-IR spectroscopy; Paris spp.

\section{INTRODUCTION}

Specific targeted quantification for quality evaluation of natural herbs has been widely accepted by official documents. It has also been applied to evaluate and discriminate different geographical origins of the samples, ${ }^{1}$ species, ${ }^{2}$ medicinal parts, ${ }^{3}$ and processing methods ${ }^{4}$ of herbal medicine in the past years. However, several bioactive compound markers hardly respond to the quality control of the medicinal plant that has multifarious constituents and pharmacological effects. For example, previous studies have reported alkaloid berberine hydrochloride with the same standard of quality control employed to Coptis chinensis as well as Phellodendron chinensis. ${ }^{5}$ Evidently, targeted quantification, as an evaluation strategy, lacks a comprehensive and integral profile. Recently, non-targeted methodology regarding spectrographic fingerprint, combined with chemometric fingerprint, has been used to evaluate herbal medicines. ${ }^{6}$ Fourier transform infrared (FT-IR) spectroscopy, especially, offers some advantages for its non-destructive, convenient and comprehensive characteristics. ${ }^{7}$

Paris (Liliaceae) is a genus presenting 24 species in the world, 19 of which are species native to China, especially in the Southwestern Yunnan Province (Figure 1). ${ }^{8}$ The rhizome of P. polyphylla var. yunnanensis (PPY) and P. polyphylla var. chinensis, known by the popular name of rhizome paridis, are described in the Chinese Pharmacopeia. ${ }^{5}$ Previous studies indicated that rhizome paridis contains steroid saponins, flavones, and aliphatic acids as its main phytochemical constituents, and it also shows anti-tumor, antimicrobial, hemostatic, anthelmintic and immune-stimulating properties. ${ }^{9,10}$ In addition, the ethnic minority of the Yunnan Province people possess a different medicinal practice for themselves. Various species have been used for a long time as folk medicine in treatments

*e-mail: ysb9-116@.com; boletus@126.com as anthelmintic and anti-tumor agents. Nowadays, rhizome paridis has been employed as material to make some Chinese patent medicine including "Yunnan Bai Yao" and "Gong Xue Ning capsule".

Rhizome paridis has a long growing period, demanding more than four years, and the current natural medicinal sources do not meet the demand for commercial productions. Therefore, substitute sources of species of rhizome paridis started being needed in recent years. Besides, Yunnan is located in the southwest of China in a region that presents a different kind of terrain, topography and climate. Most of the terrain is made of mountains and plateau regions, and the highest altitude is in the northwest of over $5000 \mathrm{~m}$, while the lowest is only about $100 \mathrm{~m}$, in the south. For PPY, it grows in most of the Yunnan Province with evergreen broad-leaved forest and pine bushes at 1400-3100 m. ${ }^{8}$ Different geographical conditions may lead to different chemical profiles for Paris, as demonstrated in the previous study which showed various content of chemical components of Paris with different geographical origins. ${ }^{11}$ Therefore, the search for Paris species of high quality with the appropriate geographical regions is necessary.

Paris saponin I (PSI), paris saponin II (PSII), paris saponin VI (PSVI) and paris saponin VII (PSVII) (Figure 2) are all documented by the Chinese Pharmacopeia. The four bioactive constituents possess the pharmacological effect of inhibiting nasopharyngeal carcinoma epithelial cell and ethanol-induced gastric lesions. ${ }^{8}, 12$ In the previous study, it was illustrated that the content of the four targeted steroid saponins varied according to the species and geographical origins. ${ }^{11,13}$ Non-targeted profile of FT-IR spectroscopy has been used to evaluate and discriminate the Paris. ${ }^{14}$ However, few researches have shown interest in the combination of targeted and non-targeted methods for the evaluation of Paris. The investigation has been concentrated mainly on PPY with respect to isolating bioactive compounds and testing the pharmacological effect. ${ }^{15,16}$ Few studies have paid attention to other species such as $P$. vietnamensis (PV), P. mairei (PM) and P. axialis (PA), whose constituents were associated with rhizome paridis, based 


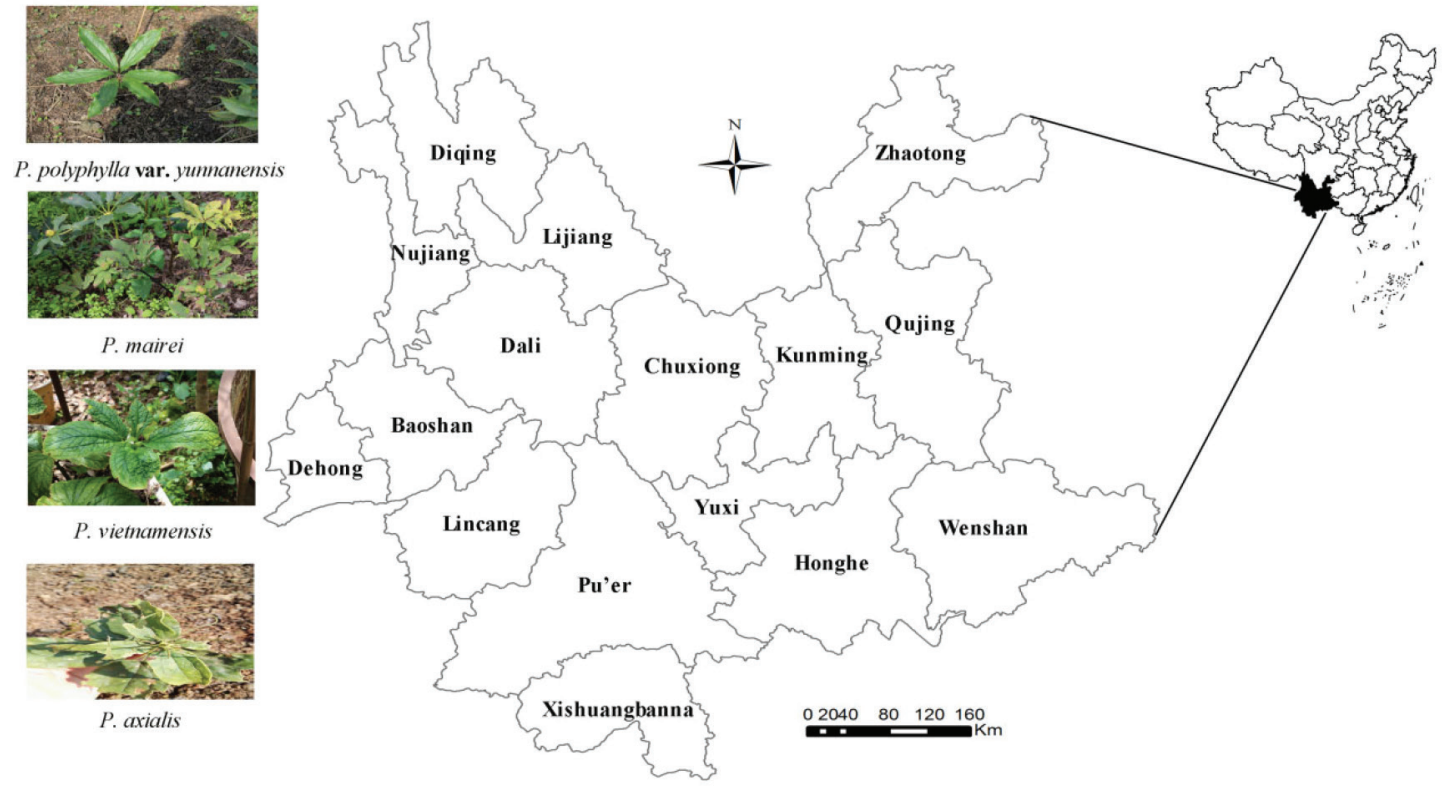

Figure 1. The detail information of genus Paris collected from Yunnan Province, southwestern China

on previous literature. ${ }^{17}$ In addition, it has been found that PPY from three regions of the Yunnan Province could be discriminated by using UHPLC and UV spectroscopy. ${ }^{18}$ However, studies dealing with the chemical composition in accurate sites have not been conducted yet.

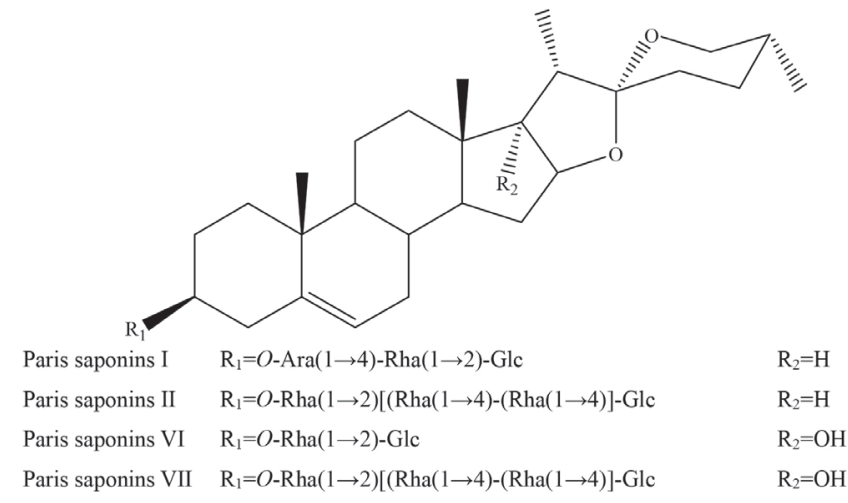

Ara $=\alpha$ - $D$-arabinofuranosyl; Rha $=\alpha-L$-rhamnopyranosyl; Glc $=\beta$ - $D$-glucopyranoside

Figure 2. Chemical structures of Paris saponins I, Paris saponins II, Paris saponins VI and Paris saponins VII

The aim of this study is to establish a rapid method based on simultaneous determination by UHPLC-UV of four bioactive constituents: PSI, PSII, PSVI and PSVII. In addition, FT-IR spectroscopy and UHPLC coupled with multivariate statistical analysis, namely supervised pattern recognition-partial least square discriminant analysis (PLS-DA) and similarity analysis, were employed to evaluate Paris including PPY, PV, PM and PA from different geographical origins. The relationship between the content of steroid saponins and Paris with different geographical origins and species was also studied.

\section{MATERIALS AND METHODS}

\section{Chemicals and reagents}

Standard compounds of PSI, PSII, PSVI and PSVII were purchased from Chengdu Must Biotechnology Co., Ltd. (Chengdu, China). The purity (>98\%) of all reference compounds were tested by UHPLC-UV. Acetonitrile and formic acid of HPLC-grade were purchased from Dikmapure Co., Ltd. (Lake Forest, MA, USA). Distilled water was prepared by Milli-Q water purification system (Bedford, MA, USA). Analytical-grade methanol was provided by Kemiou Chemical Reagent Co., Ltd. (Tianjin, China). Potassium bromide of spectroscopic grade was purchased from Fengchuan Fine Chemical Research Institute (Tianjin, China).

\section{Plant materials}

Sixty-six batches of fresh wild Paris spp. were collected in the Yunnan Province of China. Four species including PPY, PM, PV and PA were collected from Kunming, Yuxi, Wenshan, Baoshan, Chuxiong, Dehong, Honghe, Lincang, Nujiang, Xishuangbanna, Pu'er and Lijiang, and all of them were authenticated by Professor Hang Jin (Yunnan Academy of Agricultural Sciences). The detailed information is listed in Table 1. Voucher specimens were deposited at the Institute of Medicinal Plants, Yunnan Academy of Agricultural Sciences (Kunming, China). Each plant sample was washed and dried at $60{ }^{\circ} \mathrm{C}$ in the oven. The processed rhizome was pulverized and the obtained powder were passed through a 60 mesh sieve. Then, the powdered materials were stored in valve plastic bags until further analysis.

\section{Sample preparation and standard solution}

The sample $(0.10 \mathrm{~g})$ was accurately weighted and extracted with $2.00 \mathrm{~mL} \mathrm{80 \%} \mathrm{methanol} \mathrm{using} \mathrm{ultrasonication} \mathrm{for} 40 \mathrm{~min}$ at $100 \mathrm{~Hz}$. After cooling to the room temperature $\left(25^{\circ} \mathrm{C}\right)$, the extracted solution was completed with $80 \%$ methanol till the initial weight was reached. The collected supernatant which was used as the sample solution was filtered using a $0.22 \mu \mathrm{m}$ membrane filter for the UHPLC quantitative analysis.

The standard stock solutions for targeted quantification were prepared by dissolving four reference standards with the methanol to a final concentration of $1.00 \mathrm{mg} \mathrm{mL}^{-1}$. Then the stock solutions were diluted with methanol to appropriate standard samples for the further construction of calibration curves.

For the FT-IR spectroscopy, the powder of each sample $(1.50 \mathrm{mg})$ and potassium bromide $(100 \mathrm{mg}$ ) were weighed accurately and mixed evenly to press into a tablet in the agate mortar. The background of absorption of $\mathrm{CO}_{2}$ and $\mathrm{H}_{2} \mathrm{O}$ was deducted before each experiment. 
Table 1. The detail information for Paris with different species and geographical regions

\begin{tabular}{cccc}
\hline Num. & Code & Species & Site \\
\hline $1 \sim 6$ & PPY-KM1 PPY-KM6 & P. polyphylla var. yunnanensis & Kunming City \\
7 & PPY-CX1 & P. polyphylla var. yunnanensis & Yity \\
$8 \sim 10$ & PPY-YX1 PPY-YX3 & P. polyphylla var. yunnanensis & Baoshan City \\
$11 \sim 21$ & PPY-BS1 PPY-BS11 & P. polyphylla var. yunnanensis & Nujiang of Lisu Autonomous Prefecture \\
$22 \sim 26$ & PPY-NJ1 PPY-NJ5 & P. polyphylla var. yunnanensis & Licang City \\
27 & PPY-LC1 & P. polyphylla var. yunnanensis & Xishuangbanna of Dai Autonomous Prefecture \\
$28 \sim 29$ & PPY-BN1 PPY-BN2 & P. polyphylla var. yunnanensis & Dehong Autonomous Perfecture \\
30 & PPY-DH1 & P. polyphylla var. yunnanensis & Pu'er City \\
$31 \sim 32$ & PPY-PE1 PPY-PE2 & P. polyphylla var. yunnanensis & Wenshan of Zhuang and Miao Autonomous Prefecture \\
$33 \sim 35$ & PPY-WS1 PPY-WS3 & P. polyphylla var. yunnanensis & Honghe of Hani-Yi Autonomous Prefecture \\
36 & PPY-HH1 & P. polyphylla var. yunnanensis & Lijiang City \\
$37 \sim 46$ & PM-LJ1 PM-LJ10 & P. mairei & Pu'er City \\
$47 \sim 56$ & PV-PE1 PV-PE10 & P. vietnamensis & Baoshan City \\
$57 \sim 66$ & PA-BS1 PA-BS10 & P. axialis &
\end{tabular}

FT-IR spectra of each sample were registered three times after preheating for 60 min under the relative humidity of $65 \%$ at room temperature, and the average spectra were used for further analysis.

\section{Equipments}

The analysis was performed on a UHPLC system (Shimadzu, Kyoto, Japan) comprising a degasser, binary gradient pumps, UV detector, a column oven and an auto-sampler. Validated parameters were carried out as in the previous study. ${ }^{18}$ The separation was performed on a Shim-pack XR-ODS III column $(150 \times 2.0 \mathrm{~mm}, 2.2 \mu \mathrm{m})$. The flow rate was $0.35 \mathrm{~mL} / \mathrm{min}$ and the column temperature was maintained at $45{ }^{\circ} \mathrm{C}$. The mobile phase consisted of (A) $0.05 \%$ formic acid in water and (B) acetonitrile using a gradient program of $17 \% \mathrm{~B}$ for the first $1.5 \mathrm{~min}, 17-23 \% \mathrm{~B}$ for $1.5-4.0 \mathrm{~min}, 23 \% \mathrm{~B}$ for $4.0-8.7 \mathrm{~min}, 23-38 \% \mathrm{~B}$ for $8.7-18 \mathrm{~min}, 38-60 \% \mathrm{~B}$ for $18-25.6 \mathrm{~min}, 60-17 \% \mathrm{~B}$ for $25.6-28 \mathrm{~min}$, and the last $4 \mathrm{~min}$ for equilibration of the initial solvent composition. The detection wavelength was set at $203 \mathrm{~nm}$ for quantitative analysis. All samples of Paris were injected with $1 \mu \mathrm{L}$ of volume.

For the identification of the four reference standards, a Shimadzu LC-MS-8030 triple quadruple mass spectrometer operated in the electrospray ionization mode was employed, and the parameters were set as follows: the mass range in full scanning mode was at $\mathrm{m} / \mathrm{z}$ 100-1100 Da in both positive and negative ionization mode; nitrogen was the nebulizing and drying gas, with a flow rate of 3.0 and $15.0 \mathrm{~L} \mathrm{~min}^{-1}$, respectively; the interface voltage was set to $4.5 \mathrm{kV}$; and the desolvation and heat block temperatures were 250 and $400{ }^{\circ} \mathrm{C}$, respectively.

A FT-IR spectrometer (Perkin-Elmer, Norwald, CT, USA), equipped with a DTGS detector, was used to scan the samples. Each spectrum was recorded in the scanning range of $4000-400 \mathrm{~cm}^{-1}$ and a total of 64 scans with $4 \mathrm{~cm}^{-1}$ resolution. In order to obtain a better performance, multiplicative scatter correction and standard normal variate were conducted to reduce particle size effect which was related to the scattering in spectra. In addition, Savitzky-Golay filters and Wavelet compression were used to eliminate signal noise.

\section{Data analysis}

Chromatographic fingerprint analysis was conducted by Similarity Evaluation System for Chromatographic Fingerprint of Traditional Chinese Medicine (Version 2004A), Chinese Pharmacopoeia Committee. Similarity analysis of FT-IR spectra was performed by
OMINIC 8.0 (Thermo Fisher Scientific, USA). In addition, PLS-DA were carried out employing the SIMCA-P ${ }^{+} 13.0$ software (Umetrics $\mathrm{AB}$, Sweden).

\section{RESULTS AND DISCUSSION}

\section{Method validation}

The linearity of calibration curve, limit of determination (LOD), low limit of quantification (LLOQ), intra- and inter-day precision, accuracy and stability were all determined in order to investigate each compound in Paris spp. The method validation was carried out by four standard constituents in agreement with the Guidance for industry: Bioanalytical Method Validation, as specified by the FDA. ${ }^{19}$ The calibration curves were established with the stock solution of standards in methanol, being diluted with the same solvent into six appropriate concentrations. Different concentrations, from low to high, were injected into the UHPLC-UV system. The linear regression equation was obtained by plotting the peak area $\mathrm{Y}$ against the concentration $\mathrm{X}$ $\left(\mu \mathrm{gL}^{-1}\right)$. LOD and LLOQ were calculated by signal-to-noise ratio of 3 and 10, respectively. The results are listed in Table 2 . All the calibration curves showed good linearity $\left(\mathrm{R}^{2}>0.9993\right)$. LOD ranged from 11.38 to $17.56 \mu \mathrm{g} \mathrm{mL}^{-1}$, and LLOQ from 34.48 to $53.22 \mu \mathrm{g} \mathrm{mL} \mathrm{m}^{-1}$. Stability of sample solution was calculated by peak areas of analytes after storing at room temperature for $0,2,4,12$ and $24 \mathrm{~h}$. The results indicated that all analytes were stable within $24 \mathrm{~h}(\mathrm{RSD}<3.78 \%)$.

For intra- and inter-day precision, three different concentrations levels (low, medium and high quality control) of five replicates each day for three consecutive days were performed experimentally. As shown in Table 1S, RSD of intra- and inter-day variations were $\leq 3.22 \%$ and $3.76 \%$, respectively. For accuracy of the method, the recovery rate was calculated by the sample being spiked with three different concentrations $(50 \%, 100 \%$ and $150 \%$ of the known amount in sample). The formula [Recover rate $=($ detected amount - original amount) / added amount] was carried out. The results are listed in Table 2S. The range of recovery rate was from $97.17 \%$ to $101.00 \%$, and the RSD was less than $2.79 \%$.

\section{Targeted quantification for quality evaluation}

Four reference standards were identified using retention time, structure and analysis of the literature data. Diosgenin-type and pennogenin-type saponins, as the main structures, were found in 
Table 2. Calibration curve, LOD, LLOQ and stability of four standards for Paris

\begin{tabular}{|c|c|c|c|c|c|c|}
\hline analytes & regression equation & $\begin{array}{l}\text { linearity range } \\
(\mu \mathrm{g} / \mathrm{mL})\end{array}$ & $r^{2}$ & $\begin{array}{l}\text { limit of detection } \\
(\mu \mathrm{g} / \mathrm{mL})\end{array}$ & $\begin{array}{c}\text { low limit of } \\
\text { quantitation }(\mu \mathrm{g} / \mathrm{mL})\end{array}$ & stability (RSD, \%) \\
\hline PSI & $Y=169.695 X-1562.7$ & $46.66-1000$ & 0.9996 & 11.38 & 34.48 & 2.35 \\
\hline PSII & $Y=156.569 X-3293.11$ & $76.66-1000$ & 0.9994 & 17.56 & 53.22 & 3.78 \\
\hline PSVI & $Y=233.329 X-2281.26$ & $46.66-1000$ & 0.9993 & 14.67 & 44.44 & 2.96 \\
\hline PSVII & $\mathrm{Y}=179.461 \mathrm{X}-1247.42$ & $46.66-1000$ & 0.9994 & 13.68 & 41.46 & 3.34 \\
\hline
\end{tabular}

Paris. Rhamnose, arabinose and glucose were the major sugar moiety of saponins. Different features in the chemical structures could be responsible for different pharmacological effects. For instance, the structure of diosgenin-tereaglycoside (PSII) was different from pennogenin-type (PSVII) with the linkage of hydroxyl group at the $\mathrm{C}-17$. Previous studies indicated that PSII was used for treatment as an immune-stimulating and haemostatic agent, ${ }^{20,21}$ while an antifungal property was tested for PSVII. ${ }^{22}$ In this study, the different content of these structures distributed in different species and geographical origins for Paris were investigated.

\section{Quantitative analysis for different species}

The validated UHPLC-UV method was applied for the simultaneous quantification of four steroids saponins in rhizome of different species of Paris. As shown in Table 3 and Figure 3(A), the average amount of total saponains including PSI, PSII, PSVI and PSVII of PA (43.36 $\mathrm{mg} \mathrm{g}^{-1}$ ) collected in Baoshan City of the Yunnan Province was the highest in comparison with other species, and the lowest was PM (11.60 $\mathrm{mg} \mathrm{g}^{-1}$ ) collected from Lijiang City. As an official medicinal material, the average content of total saponins for PPY could reach up to $22.72 \mathrm{mg} \mathrm{g}^{-1}$, which were $6 \mathrm{mg} \mathrm{g}^{-1}$ above the threshold value according to the Chinese Pharmacopeia (2015 edition). In addition, all employed species in this study were qualified as the current document for evaluation of Paris. Each individual had a significant variety of content in the same species. Taking PPY as an example: the highest total saponins in PPY-BN2 $\left(85.25 \mathrm{mg} \mathrm{g}^{-1}\right)$ collected from Xishuangbanna was 17.83 times higher than that in PPY-BS9 (4.78 $\mathrm{mg} \mathrm{g}^{-1}$ ) collected from Baoshan. The total saponin in PM-LJ6 (23.12 $\mathrm{mg} \mathrm{g}^{-1}$ ) was 13.68 times higher than that in PM-LJ10 $\left(1.69 \mathrm{mg} \mathrm{g}^{-1}\right)$ for the same species and geographical origins as well, which was markedly different. The results indicated that the secondary metabolites of the plant were correlated with genetic variations and growing conditions. The species and geographical regions, as in the previous study, had an influence on the accumulation of metabolites for Gentianaceae species. ${ }^{23}$ For each saponins, PSI had the highest content in most of the individuals, and PSVI was too low to be detected, except for PPY-NJ2 (Nujiang), PPY-BN2 (Xishuangbanna), PPY-PE1 and PPY-PE2 (Pu'er). In addition, the observed content of PSII was higher than that of PSVII. At last, it could be found that the content of diosgenin-type was higher than that of pennogenin-type. The content of each structure in different species was investigated, since there are various bioactive effects for each different structure. The results are listed in Table $3 \mathrm{~S}$ and Figure 3(B). The percentage of each compound against the total content for four species was displayed. The data showed that PSI was the main metabolite in PA while PSII and PSVI were in PPY. The highest content ratio of PSVII was detected in PM. The highest percentage of diosgenin-type, including PSI and PSII, were found in PA. On the contrary, the percentage of pennogenin-type, including PSVI and PSVII, was the highest in PM.

Quantitative analysis of different geographical origins for PPY

Table 3 and Figure 3(C) show the average content of total saponins for PPY of different cities of the Yunnan Province. The highest value in PPY-BN from Xishuangbanna (61.44 $\left.\mathrm{mg} \mathrm{g}^{-1}\right)$ was 5.03 times higher than that in PPY-CX from Chuxiong $\left(12.22 \mathrm{mg} \mathrm{g}^{-1}\right)$. Interestingly, samples collected from southern Yunnan Province including Lincang, Xishuangbanna, Dehong, Pu'er, Wenshan and Honghe had higher average content (all above $24.87 \mathrm{mg} \mathrm{g}^{-1}$ ) when compared with other regions. PPY from different sites of the Yunnan Province were qualified in comparison with Chinese Pharmacopeia (2015 editor). A significant variety of total saponins for each sample of the same area were displayed. For example, the total saponins of PPY-BS1 (29.92 $\mathrm{mg} \mathrm{g}^{-1}$ ) was 6.26 times higher than that of PPY-BS9 $\left(4.78 \mathrm{mg} \mathrm{g}^{-1}\right)$ from Baoshan. For each saponin of sample, the order of content, from highest to lowest, was PSI, PSII, PSVII and PSVI. The content of PSI that came from the same area varied remarkably, being 11.16 times higher in PPY-BS1 (24.55 $\left.\mathrm{mg} \mathrm{g}^{-1}\right)$ than in PPY-BS9 $\left(2.20 \mathrm{mg} \mathrm{g}^{-1}\right)$. This indicated that the geographical condition had an effect on the secondary metabolites of the medicinal plant. To investigate the detailed chemical profile for different sites of PPY, the percentage of each saponins versus total saponins is displayed in Table 3S and Figure 3(D). As it can be seen, the main metabolites of PSI, PSII, PSVI and PSVII were in Baoshan (PYY-BS), Kunming (PPY-KM), Pu'er (PPY-PE) and Yuxi (PPY-YX), respectively. Samples of Chuxiong (PPY-CX) and Yuxi (PPY-YX) had the highest percentage of diosgenin-type and pennogenin-type, respectively. Targeted quantification of bioactive markers, to some extent, could evaluate the different species of Paris and geographical origins. However, some trace of bioactive compounds might be neglected. In addition, the rhizomes of Paris have been extracted and the solutions have been employed for medicinal purposes in China for thousands of years. The relationship between chemical profile and geographical regions and species has not been illustrated either. Evidently, there is a need to establish a comprehensive and rapid methodology for the evaluation Paris of different areas and species.

\section{Non-targeted evaluation for Paris spp extracts}

\section{Similarity analysis}

Fingerprint has been widely used to evaluate herbal medicine to perform the complete chemical profile. In this study, similarity analysis including chromatographic and spectrographic methods were employed to evaluate Paris. The typical chromatography for Paris in different species (A) and geographical origins (B) are shown in Figure 4 . The average chromatogram of sixty-six batches of sample was treated as the standard characteristic fingerprint. The correlation coefficient was calculated by each chromatogram of sample against the average chromatogram. As shown in Table $4 \mathrm{~S}$, the value of all samples ranged from 0.37 to 0.59 . The results were associated with quantitative analysis of the various chemical profiles displayed for Paris of different sites and species. Figure 4 shows the FT-IR spectra of sample in different species (C) and geographical origins (D). Similar position and shape of peak including 3400, 2930, 1700, 1400, $1150,1049,930$ and $860 \mathrm{~cm}^{-1}$ were found in most of the samples. The peak at $3400 \mathrm{~cm}^{-1}$ was assigned to the stretching vibration peak of $\mathrm{O}-\mathrm{H}$ in sugar moiety. The wavelength at $2930 \mathrm{~cm}^{-1}$ was due to 
Table 3. The content of PSI, PSII, PSVI and PSVII for Paris in different species and geographical origins

\begin{tabular}{|c|c|c|c|c|c|c|c|c|c|c|c|}
\hline code & $\begin{array}{c}\text { PSI } \\
\left(\mathrm{mg} \mathrm{g}^{-1}\right)\end{array}$ & $\begin{array}{c}\text { PSII } \\
\left(\mathrm{mg} \mathrm{g}^{-1}\right)\end{array}$ & $\begin{array}{c}\text { PSVI } \\
\left(\mathrm{mg} \mathrm{g}^{-1}\right)\end{array}$ & $\begin{array}{c}\text { PSVII } \\
\left(\mathrm{mg} \mathrm{g}^{-1}\right)\end{array}$ & $\begin{array}{l}\text { total saponin } \\
\left(\mathrm{mg} \mathrm{g}^{-1}\right)\end{array}$ & code & $\begin{array}{c}\text { PSI } \\
\left(\mathrm{mg} \mathrm{g}^{-1}\right)\end{array}$ & $\begin{array}{c}\text { PSII } \\
\left(\mathrm{mg} \mathrm{g}^{-1}\right)\end{array}$ & $\begin{array}{c}\text { PSVI } \\
\left(\mathrm{mg} \mathrm{g}^{-1}\right)\end{array}$ & $\begin{array}{c}\text { PSVII } \\
\left(\mathrm{mg} \mathrm{g}^{-1}\right)\end{array}$ & $\begin{array}{l}\text { total saponin } \\
\left(\mathrm{mg} \mathrm{g}^{-1}\right)\end{array}$ \\
\hline PPY-KM1 & 7.19 & 7.02 & ND & ND & 14.21 & PPY-WS2 & 21.84 & 14.59 & ND & 6.54 & 42.97 \\
\hline PPY-KM2 & 8.41 & 11.83 & ND & 1.80 & 22.04 & PPY-WS3 & 17.87 & 16.58 & 2.10 & 7.74 & 44.29 \\
\hline PPY-KM3 & 6.14 & 4.97 & $\mathrm{ND}$ & ND & 11.11 & PPY-HH1 & 14.55 & 9.25 & ND & 5.21 & 29.01 \\
\hline PPY-KM4 & 6.50 & 3.29 & ND & ND & 9.79 & PM-LJ1 & 0.65 & ND & ND & 2.32 & 2.97 \\
\hline PPY-KM5 & 7.29 & 8.25 & ND & 2.80 & 18.35 & PM-LJ2 & 2.84 & 1.61 & ND & 2.38 & 6.84 \\
\hline PPY-KM6 & 4.33 & 3.23 & ND & 2.13 & 9.69 & PM-LJ3 & 2.64 & 2.42 & ND & 2.60 & 7.66 \\
\hline PPY-CX1 & 7.10 & 5.11 & ND & ND & 12.22 & PM-LJ4 & 5.97 & 2.90 & ND & 1.58 & 10.45 \\
\hline PPY-YX1 & 2.36 & 3.11 & ND & 1.81 & 7.28 & PM-LJ5 & 10.99 & 3.22 & ND & 4.42 & 18.64 \\
\hline PPY-YX2 & 21.65 & 1.64 & ND & 13.74 & 37.03 & PM-LJ6 & 10.53 & 7.37 & ND & 5.22 & 23.12 \\
\hline PPY-YX3 & 0.82 & 1.28 & ND & 3.58 & 5.68 & PM-LJ7 & 5.10 & 3.33 & ND & 5.20 & 13.62 \\
\hline PPY-BS1 & 24.55 & 4.41 & ND & 0.95 & 29.92 & PM-LJ8 & 7.30 & 3.54 & ND & 3.15 & 13.98 \\
\hline PPY-BS2 & 5.91 & 2.24 & ND & 1.22 & 9.37 & PM-LJ9 & 12.15 & 4.88 & ND & ND & 17.03 \\
\hline PPY-BS3 & 6.10 & 2.79 & ND & 1.14 & 10.03 & PM-LJ10 & 0.76 & ND & ND & 0.94 & 1.69 \\
\hline PPY-BS4 & 12.89 & 5.08 & ND & ND & 17.97 & PV-PE1 & 0.71 & 2.25 & ND & 1.10 & 4.06 \\
\hline PPY-BS5 & 2.73 & 1.60 & ND & 1.59 & 5.91 & PV-PE2 & 28.14 & 7.43 & ND & 2.92 & 38.49 \\
\hline PPY-BS6 & 14.43 & 4.07 & ND & 1.04 & 19.54 & PV-PE3 & 1.25 & 2.47 & ND & 1.13 & 4.85 \\
\hline PPY-BS7 & 15.81 & 6.08 & ND & 1.64 & 23.53 & PV-PE4 & 15.76 & 5.04 & ND & 2.39 & 23.19 \\
\hline PPY-BS8 & 3.71 & 2.12 & ND & 1.17 & 7.00 & PV-PE5 & 3.59 & 1.50 & ND & ND & 5.09 \\
\hline PPY-BS9 & 2.20 & 1.65 & ND & 0.93 & 4.78 & PV-PE6 & 13.42 & 4.57 & ND & 1.81 & 19.80 \\
\hline PPY-BS10 & 7.07 & 2.82 & ND & 1.25 & 11.14 & PV-PE7 & 19.54 & 4.34 & ND & 1.58 & 25.46 \\
\hline PPY-BS11 & 12.18 & 10.40 & ND & ND & 22.58 & PV-PE8 & 1.13 & 2.30 & ND & 1.34 & 4.76 \\
\hline PPY-NJ1 & 16.98 & 5.60 & ND & 3.32 & 25.90 & PV-PE9 & 16.98 & 6.59 & ND & 0.97 & 24.54 \\
\hline PPY-NJ2 & 19.48 & 26.54 & 2.37 & 5.15 & 53.54 & PV-PE10 & 12.56 & 3.37 & ND & 1.46 & 17.39 \\
\hline PPY-NJ3 & 11.35 & 8.99 & ND & 2.35 & 22.69 & PA-BS1 & 60.04 & 4.45 & ND & ND & 64.49 \\
\hline PPY-NJ4 & 7.69 & 10.67 & ND & 1.72 & 20.08 & PA-BS2 & 39.79 & 9.70 & ND & ND & 49.48 \\
\hline PPY-NJ5 & 9.96 & ND & ND & $\mathrm{ND}$ & 9.96 & PA-BS3 & 35.42 & 5.24 & ND & ND & 40.66 \\
\hline PPY-LC1 & 27.32 & 4.74 & ND & 12.25 & 44.31 & PA-BS4 & 25.37 & 5.21 & ND & ND & 30.58 \\
\hline PPY-BN1 & 20.77 & 11.93 & ND & 4.93 & 37.62 & PA-BS5 & 41.01 & 2.91 & ND & ND & 43.92 \\
\hline PPY-BN2 & 42.41 & 21.18 & 9.83 & 11.83 & 85.25 & PA-BS6 & 23.57 & 7.63 & ND & ND & 31.19 \\
\hline PPY-DH1 & 13.61 & 7.57 & ND & 3.69 & 24.87 & PA-BS7 & 23.72 & 9.00 & ND & ND & 32.72 \\
\hline PPY-PE1 & 7.97 & 4.65 & 2.04 & 6.88 & 21.54 & PA-BS8 & 47.21 & 8.41 & ND & ND & 55.62 \\
\hline PPY-PE2 & 13.36 & 10.07 & 3.80 & 6.05 & 33.28 & PA-BS9 & 43.51 & 5.63 & ND & ND & 49.14 \\
\hline PPY-WS1 & 1.51 & 3.90 & ND & 8.14 & 13.56 & PA-BS10 & 25.54 & 9.30 & ND & 0.99 & 35.83 \\
\hline
\end{tabular}

"ND" Compounds were not detected.

the stretching of the methylene group. Absorption band at around $1700 \mathrm{~cm}^{-1}$ corresponding to bending $\mathrm{C}=\mathrm{O}$ stretching was related to steroid saponins and flavonoids in Paris. The peak at $1400 \mathrm{~cm}^{-1}$ was assigned to the plane deviational vibrations of the methyl group. It is shown in Figure 5 that FTIR spectrum of paris saponins absorption band at 1050, 920 and $860 \mathrm{~cm}^{-1}$ were due to the steroidal saponins skeletal vibration. ${ }^{24}$ It was similar to the chromatographic method, in which the correlation coefficient was calculated by comparing each sample to the mean spectrum. The results indicated that value of all samples were above 0.91 . This implies that all Paris of different geographical origins and species had similar chemical absorbance band. Similarity analysis based on chromatographic and spectrographic methods had illustrated the chemical profiles of Paris. However, the relationship between different sites and species had not been investigated. An integrated and convenient method is necessary to be established for the next study.
Principal component analysis (PCA)

PCA is an unsupervised method that can concentrate the multidimensional data into a two-dimensional matrix. It can be used to respond to the chemical information of different samples. As shown in Figure 6A, sample of different species could not be separated well relating to the presence of PA. In Figure 6B, PPY collected from different regions could hardly be distinguished either. Therefore, an appropriate model for discrimination in the different species and regions was necessary.

Partial least squares discriminant analysis

As a supervised method, partial least squares discriminant analysis (PLS-DA) is used to classify a group of samples as belonging or not belonging to a specific class. ${ }^{25} \mathrm{~A}$ further analysis of PLS-DA model, the variable importance of projection (VIP), was used to select variables. ${ }^{26} \mathrm{~A}$ variable was treated as important to the PLS-DA 


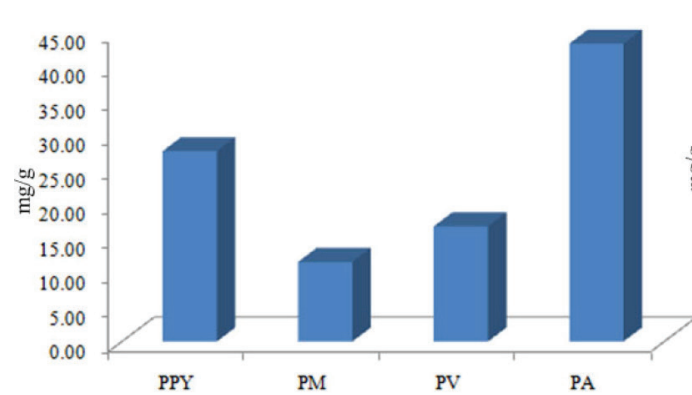

A

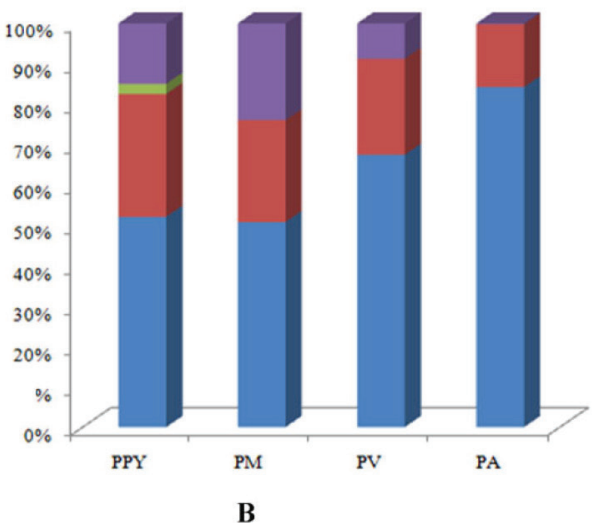

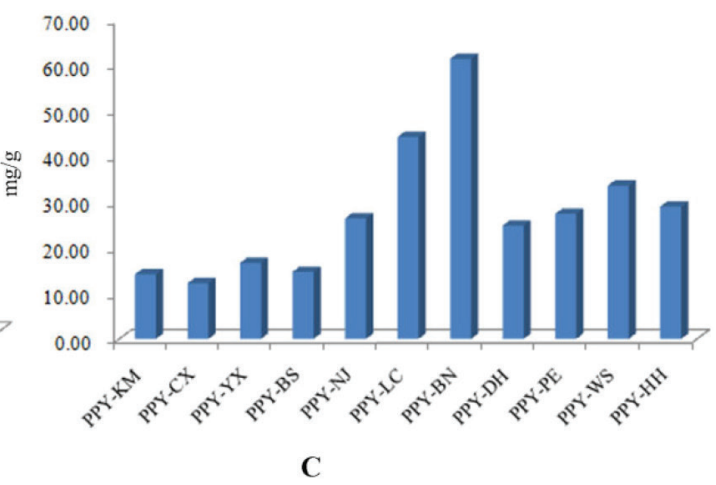

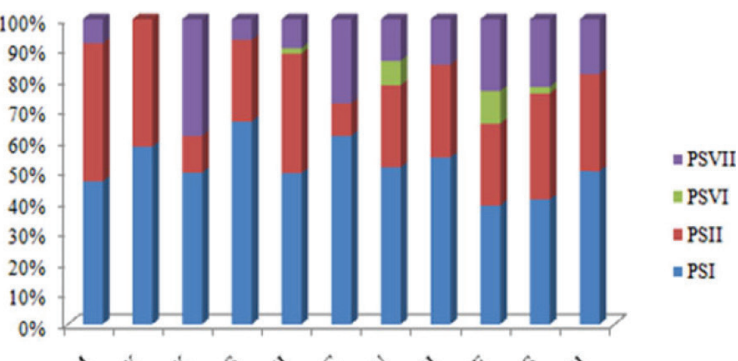

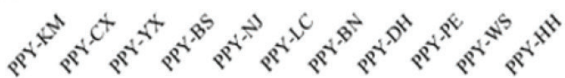

D

Figure 3. Content of the average total saponins for PPY, PM, PV and PA (A); Percentage of each compound against the total saponins in PPY, PM, PV and $P A(B)$; Content of the average total saponins for PPY with different geographical regions $(C)$; Percentage of each compound against the total saponins in PPY with different geographical regions $(D)$

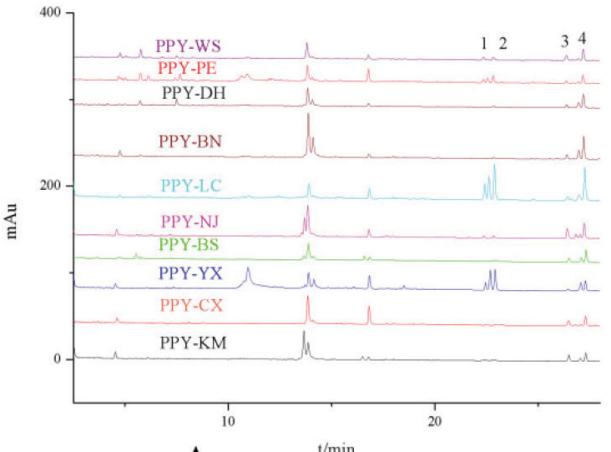

A $\quad t / m$

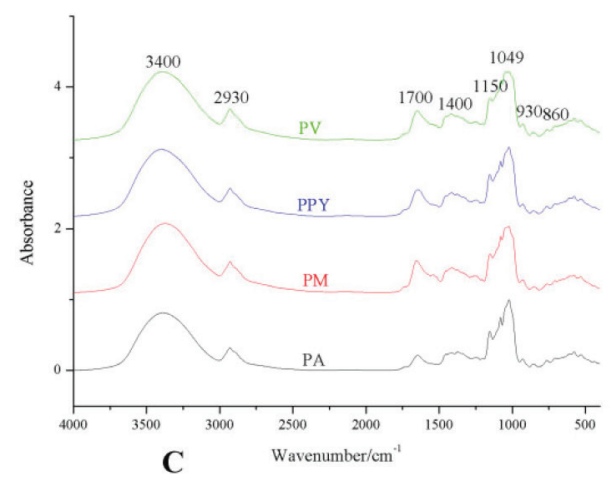

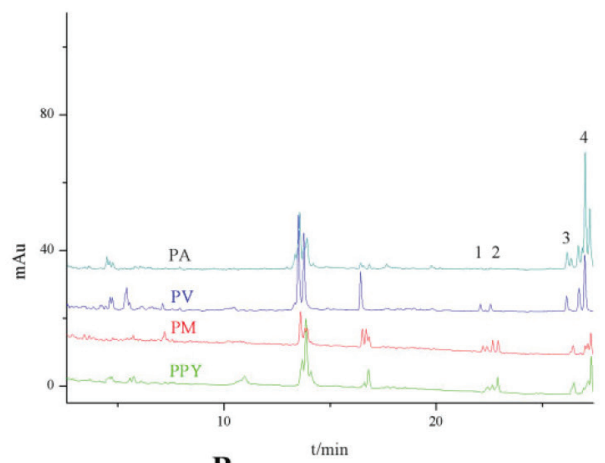

B

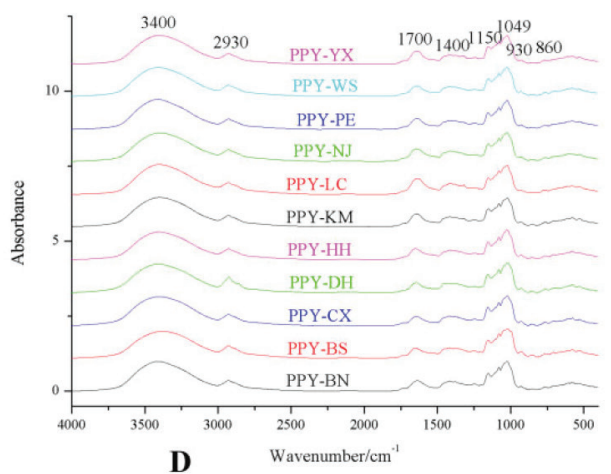

Figure 4. Typical chromatography for Paris in different species (A) and geographical origins (B); FT-IR spectra of sample in different species (C) and geographical origins (D). "1, 2, 3 and 4" were responsible for the PSVII, PSVI, PSII and PSI, respectively

model when the VIP was beyond $1.0 .^{27}$ In this study, PLS-DA based on FT-IR spectra was used to investigate Paris of different species and geographical origins.
Discrimination and evaluation of Paris spp.

As shown in Figure 7(A), a two-dimensional score scatter plot of $95 \%$ confidence ellipse is displayed. Four species (PPY, PM, 


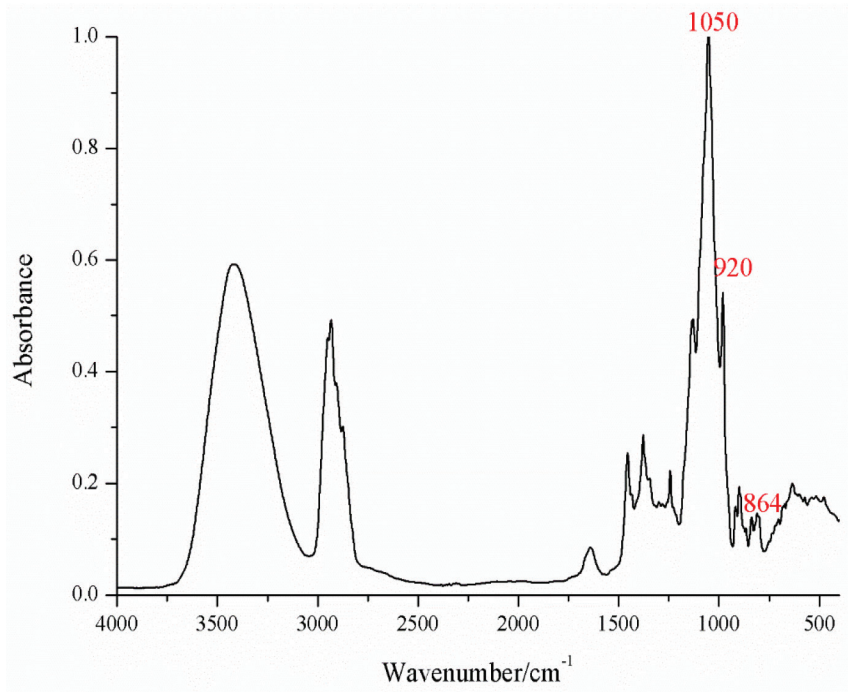

Figure 5. FTIR spectra of paris saponins
PV and PA) were investigated based on the data of FT-IR spectra. Evidently, all the PA samples in Baoshan City of the Yunnan Province were separated from the other species and geographical origins. As in the quantitative analysis, the content of PA was considerably higher than that of other species. Interestingly, two PPY, PPY-BS1 (11) and PPY-BS3 (13), collected from Baoshan City, were similar to the PA sample. This proximity implied that the geographical condition had an effect on the chemical profile of Paris. However, the other sample from Baoshan City was far away from PA. Therefore, it could be noted that species was more related to chemical characteristics than geographical sites, because the distance of the same site with different species was longer than that of the same species with different geographical origins. An outlier was found with PM collected from Lijiang, demonstrating that the chemical characteristic of PM-LJ4 (40) was different from the others. In addition, the spot of PM was scattered in the negative principal component one. The similar result was in agreement with the quantitative analysis in which the chemical constituent varied notably for each sample. For PV collected from Pu'er region,
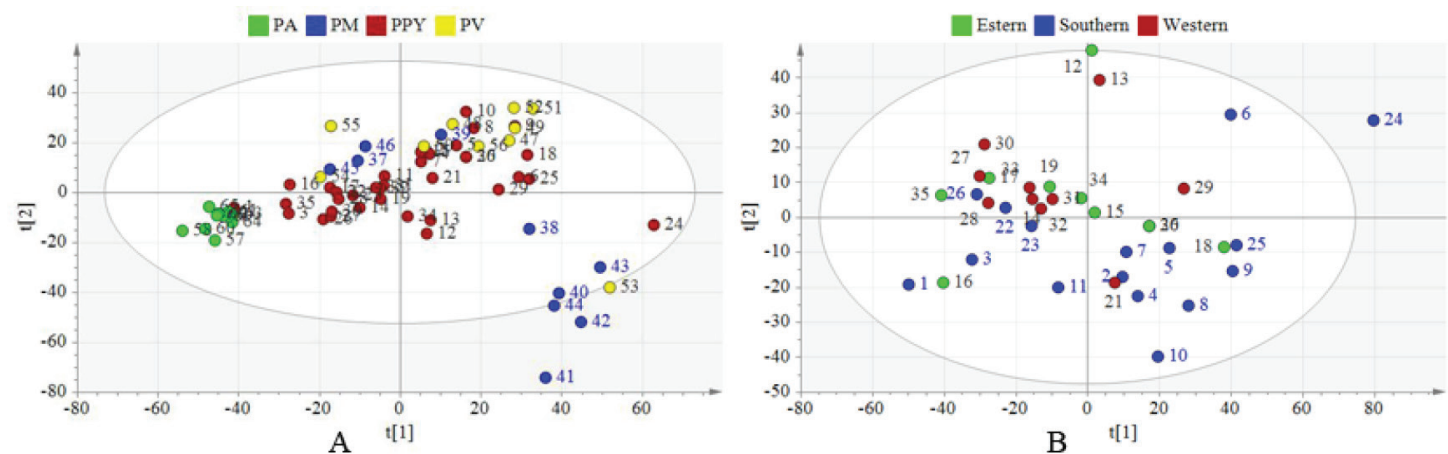

Figure 6. Two-dimension score plot of PLS-DA based on FT-IR spectroscopy for PPY, PM, PV and PA (A); Two-dimension score plot of PLS-DA for different geographical origins $(B)$
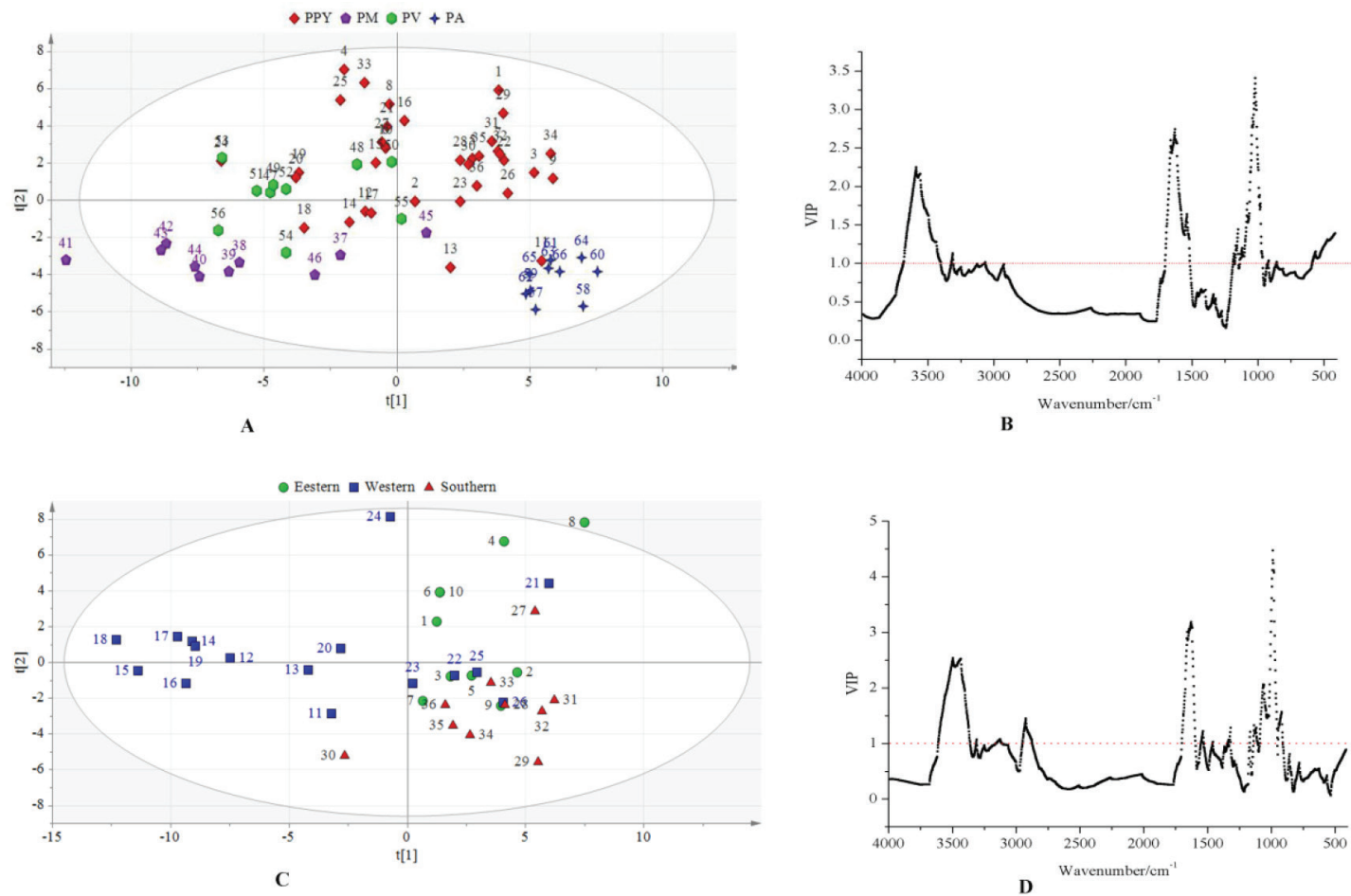

Figure 7. Two-dimension score plot of PLS-DA based on FT-IR spectroscopy for PPY, PM, PV and PA (A); VIP score plot for PPY, PM, PV and PA (B); Two-dimension score plot of PLS-DA for different geographical origins $(C)$; VIP score plot for different geographical origins $(D)$ 
the dispersive distribution was displayed as well. However, the relationship between PV and PPY was closer than other species, especially PV-PE2 (48), PV-PE4 (50) and PV-PE9 (55), almost belonging to the PPY group. As an official medicinal herb, most samples with PPY collected from different sites could be classified into one group. The scattered spots indicated that geographical condition could affect the chemical compound in PPY. VIP was used to select the important variable for the discrimination of Paris from different species. It is shown in Figure 7(B) that the VIP scores of four wavebands including $3683-3401 \mathrm{~cm}^{-1}, 1700-1513 \mathrm{~cm}^{-1}, 1186-964 \mathrm{~cm}^{-1}$ and $586-414 \mathrm{~cm}^{-1}$ were responsible for $96.5 \%$ of values above 1 . The wavelength of $3683-3401 \mathrm{~cm}^{-1}$ was generally due to the stretching vibration peaks of $\mathrm{O}-\mathrm{H}$ in sugar moieties. The absorption band that ranged from 1700 to $1513 \mathrm{~cm}^{-1}$ were potentially related to $\mathrm{C}=\mathrm{O}$ and $\mathrm{C}=\mathrm{C}$ stretching of steroid saponins, flavonoids and fatty acids. In addition, according to previous study, the peak at 1150, 1049 and $960 \mathrm{~cm}^{-1}$ were assigned to steroid saponins. Therefore, it indicated that the content of steroid saponins might be related to different species of Paris.

\section{Discrimination and evaluation of PPY in different geographical origins}

Two-dimension score plots were generated for PPY of different geographical origins. As seen in Figure 7(C), PPY collected from eastern, western and southern regions of the Yunnan Province were divided into three parts. PPY in the eastern regions were located in the positive principal component one, except for PPY-YX1 (8), which was an outlier. The majority of PPY collected in the Baoshan and Nujiang western region were listed in the positive and negative principal component. The dispersive spots had various geographical conditions such as the soil, climate and altitude in the western region of the Yunnan Province. In addition, most of the samples in the southern region were loaded in the positive principal component one. As it can be seen, some samples overlapped all three regions. These data implied that chemical profile with PPY of different regions might be affected by other factors. VIP based on PPY of different regions is shown in Figure 7(D). VIP of three wavebands including $3610-3309 \mathrm{~cm}^{-1}, 1700-1531 \mathrm{~cm}^{-1}$ and $1168-910 \mathrm{~cm}^{-1}$ were above 1 , which were selected as important variables for discrimination of PPY of different geographical origins. Those absorption bands were due to steroidal saponins skeletal vibration according to the previous VIP analysis of Paris with different species.

\section{CONCLUSIONS}

In this investigation, a method was developed and validated for the evaluation of different species and geographical origins using targeted quantitative analysis and non-targeted FT-IR spectroscopy. Targeted quantitative analysis demonstrated that the content of diosgenin-type saponin was higher than that of pennogenin-type for Paris of different species and sites. Non-targeted analysis indicated that the relationship of PPY and PV was closer than that of other species. According to the VIP analysis, the steroid saponins were related to different species and geographical origins of Paris. This could provide a fundamental construction for further investigation of Paris.

\section{SUPPLEMENTARY MATERIAL}

Tables 1S-4S are freely available at http://quimicanova.sbq.org.br, in PDF format.

\section{ACKNOWLEDGEMENTS}

This work was supported by the National Natural Science Foundation of China (No. 81460584) and Special Fund for Agroscientific Research in the Public Interest (No. 201303117).

\section{REFERENCES}

1. Zhao, L.; Zhao, H.; Zhao, X.; Kong, W.; Hu, Y.; Yang, S.; Yang, M.; Phytochem. Analysis 2016, 27, 168.

2. Tao, W.; Li, J.; Guo, S.; Qian, D.; Shang, E.; Su, D.; Duan, J.; J. Sep. Sci. 2016, 39, 473.

3. Pan, Q.; Saiman, M. Z.; Mustafa, N. R.; Verpoorte, R.; Tang, K.; J. Chromatogr. B 2016, 1014, 10

4. Cao, G.; Wu, X.; Li, Q.; Cai, H.; Cai, B.; Zhu, X.; J. Sep. Sci. 2015, 38, 390.

5. The State Pharmacopoeia Commission Chinese Pharmacopoeia, Beijing, 2015.

6. Peets, P.; Leito, I.; Pelt, J.; Vahur, S.; Spectrochim. Acta A 2017, 173, 175.

7. Wang, P.; Yu, Z.; J. Pharmaceut. Anal. 2015, 5, 277.

8. Wei, J. C.; Gao, W. Y.; Yan, X. D.; Wang, Y.; Jing, S. S.; Xiao, P. G.; Chem. Biodivers. 2014, 11, 1277.

9. Zhang, J. Y.; Wang, Y. Z.; Zhao, Y. L.; Yang, S. B.; Zuo, Z. T.; Yang, M. Q.; J. Asian Nat. Prod. Res. 2011, 13, 670.

10. Li, H.; The genus Paris (Trilliaceae); Science Press: Beijing, 1998.

11. Zhao, Y.; Zhang, J.; Yuan, T.; Shen, T.; Hou, Y.; Yang, S.; Wang, Y.; Jin, H.; Spectrosc. Spectral Anal 2014, 34, 1831.

12. Chen, Y. S.; He, Y.; Chen, C.; Zeng, Y.; Xue, D.; Wen, F. Y.; Wang, L.; Zhang, H.; Du, J. R.; Steroids 2014, 83, 39.

13. Chen, P.; Jin, H.; Sun, L.; Ma, S.; J. Sep. Sci. 2016, 39, 3550.

14. Zhao, Y.; Zhang, J.; Yuan, T.; Shen, T.; Li, W.; Yang, S.; Hou, Y.; Wang, Y.; Jin H.; PLoS One 2014, 9, e89100.

15. Wen, Y. S.; Ni, W.; Qin, X. J.; Yan, H.; Chen, C. X.; Hua, Y.; Cheng, Y. C.; He, L.; Liu, H.Y.; Phytochem. Lett. 2015, 12, 31

16. Man, S.; Fan, W.; Gao, W.; Li, Y.; Wang, Y.; Liu, Z.; Li, H.; J. Ethnopharmacol. 2014, 151, 407.

17. Yang, Y.; Zhang, J.; Zhang, J. Y.; Wang, Y.; Chin. Tradit. Herb Drugs 2016, 47, 3301.

18. Yang, Y.; Jin, H.; Zhang, J.; Zhang, J. Y.; Wang, Y.; J. Nat. Med. 2017, $71,148$.

19. Food and Drug Administration. Guidance for industry: bioanalytical method validation, available at https://www.fda.gov/downloads/drugs/ guidances/ucm070107.pdf, accessed February 2019.

20. Guo, L.; Su, J.; Deng, B. W.; Yu, Z. Y.; Kang, L. P.; Zhao, Z. H.; Shan, Y. J.; Shen, J. P.; Ma, B. P.; Cong, Y. W.; Hum. Reprod. 2008, 23, 964

21. Zhang, X.; Cui, Y.; Huang, J.; Zhang, Y.; Nie, Z.; Wang, L.; Yan, B.; Tang, Y.; Liu, Y.; Bioorg. Med. Chem. Lett. 2007, 17, 2408.

22. Deng, D.; Lauren, D. R.; Cooney, J. M.; Jensen, D. J.; Wurms, K. V.; Upritdard, J. E.; Cannon, R. D.; Wang, M. Z.; Li, M. Z.; Planta Med. 2008, 74, 1397.

23. Pan, Y.; Zhang, J.; Zhao, Y. L.; Wang, Y. Z.; Jin, H.; Phytocheml. Analysis 2017, 27, 158

24. Singh, S. B.; Thakur, R. S.; Schulten, H. R.; Phytochemistry 1980, 21, 2925.

25. Li, Y.; Zhang, J.; Jin, H.; Liu, H.; Wang, Y.; Spectrochim. Acta A. 2016, 165,61

26. Farrés, M.; Platikanov, S.; Tsakovski, S.; Tauler, R.; J. Chemom. 2015, 29,528 .

27. Afanador, N. L.; Tran, T. N.; Buydens, L. M. C.; Anal. Chim. Acta 2013, 768,49 . 